\title{
The effect of foliation orientation on the inferred rotation axes and rotation angles of rotated porphyroblasts
}

\author{
R.L.M. VISSERS \\ Department of Structural and Applied Geology, Institute of Earth Sciences, Budapestlaan 4, Postbus 80.021, \\ 3508 TA Utrecht (The Netherlands)
}

(Received January 21, 1986; revised version accepted November 24, 1986)

\begin{abstract}
Vissers, R.L.M., 1987. The effect of foliation orientation on the inferred rotation axes and rotation angles of rotated porphyroblasts. Tectonophysics, 139: 275-283.

The rotation axes and rotation angles inferred from the intersection of inclusion fabrics in rotated porphyroblasts and the external foliation are apparent axes and apparent angles whose orientation and magnitude are strongly influenced by the initial orientation of the foliation with respect to the geometry of the deformation. Spherical porphyroblasts may show apparent angles of rotation consistently smaller than the corresponding true rotation angles and apparent rotation axes oriented at a distinct angle with the true axis of rotation. For nonspherical porphyroblasts this effect may lead to a scatter of apparent rotation axes. Sections of a rock intersecting the foliation through the resulting sector of apparent axes will show apparent forward and apparent backward rotations, even when the foliation remains stationary throughout the deformation and all porphyroblasts rotate with the same sense of true rotation. This effect holds the key to one possible explanation of apparent reversals observed in some paracrystalline rotated porphyroblasts.
\end{abstract}

\section{Introduction}

Naturally deformed metamorphic rocks often contain porphyroblasts of metamorphic minerals with inclusion trails, which delineate a planar internal fabric oriented at a distinct angle to the foliation in the rock. In a number of cases such internal structure can be traced into the external foliation (Fig. 1), which clearly demonstrates that the porphyroblasts did rotate with respect to the surrounding matrix, in response to a deformation posterior to porphyroblast growth (Zwart, 1962; Spry, 1969). Another conspicuous microstructure often noted is the double-spiral or snowball pattern of inclusions in syntectonic porphyroblasts (Fig. 2), recording rotation caused by deformation synchronous with porphyroblast growth (Zwart,
1962; Powell and Treagus, 1967, 1970; Rosenfeld, 1970; Schoneveld, 1977, 1979). Various workers have made attempts to relate the amount of rotation of such porphyroblasts to the magnitude of the strain accumulated during rotation (Schmidt, 1918; Ramsay, 1962; Rosenfeld, 1970; Ghosh and Ramberg, 1976; Schoneveld, 1979) and both simple shear, pure shear, and a combination of these have been shown to be capable of producing these structures.

An important geometrical element in such analyses is the orientation of the rotation axis. In the case of rotational fabrics in syntectonic porphyroblasts, this orientation can be deduced from the three-dimensional geometry of the inclusion pattern (Powell and Treagus, 1967, 1970; Schoneveld, 1979). In contrast, postcrystalline 


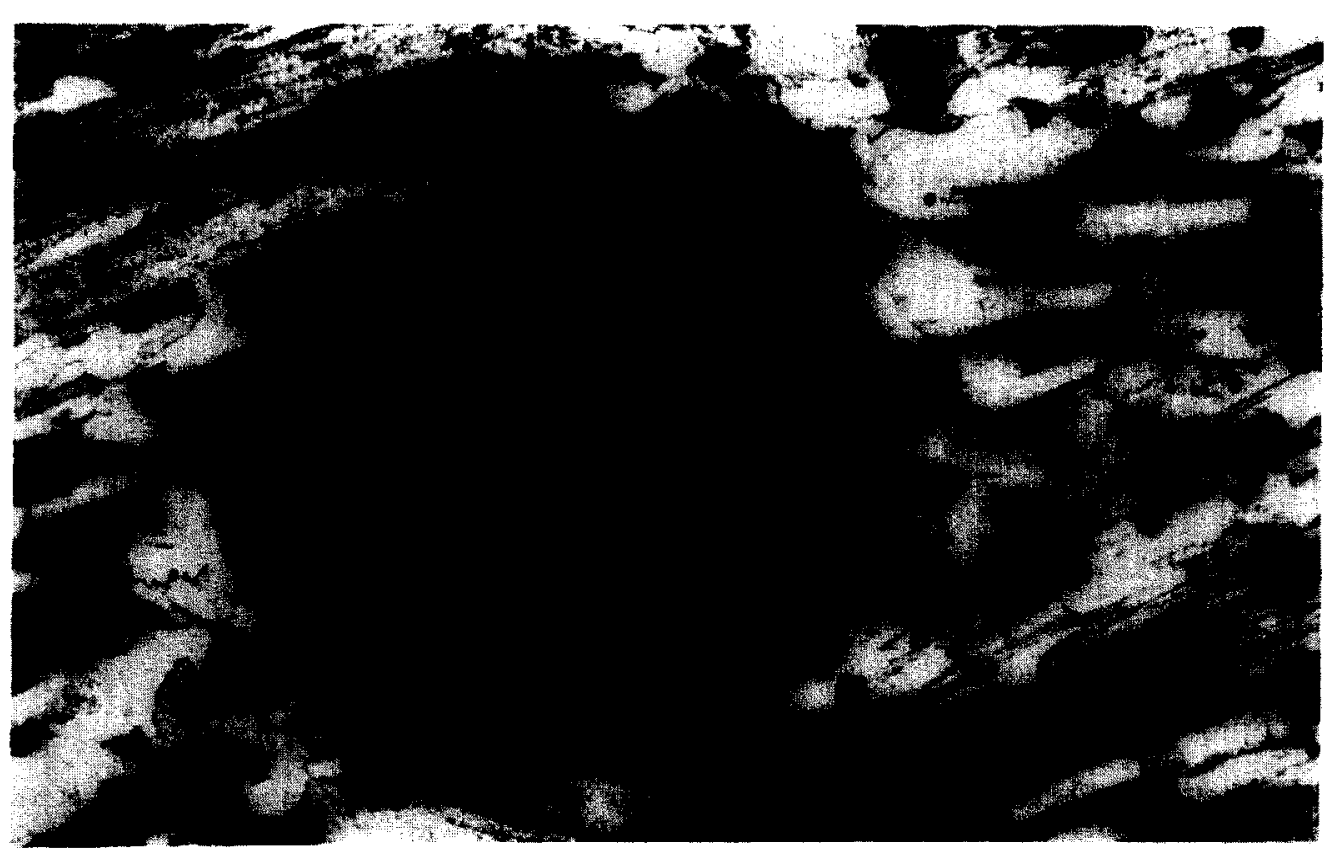

Fig. 1. Straight inclusion pattern in garnet porphyroblast grown prior to rotation (Dikanäs schist, Västerbotten, Sweden).

rotated porphyroblasts do not record the rotation axis without ambiguity. The models presented in the literature commonly discuss rotation of spherical and nonspherical objects in a viscous medium undergoing plane deformation with a component of simple shear parallel to or at an angle with a passive marker representing a pre-existing foliation. However, a restriction is inex-

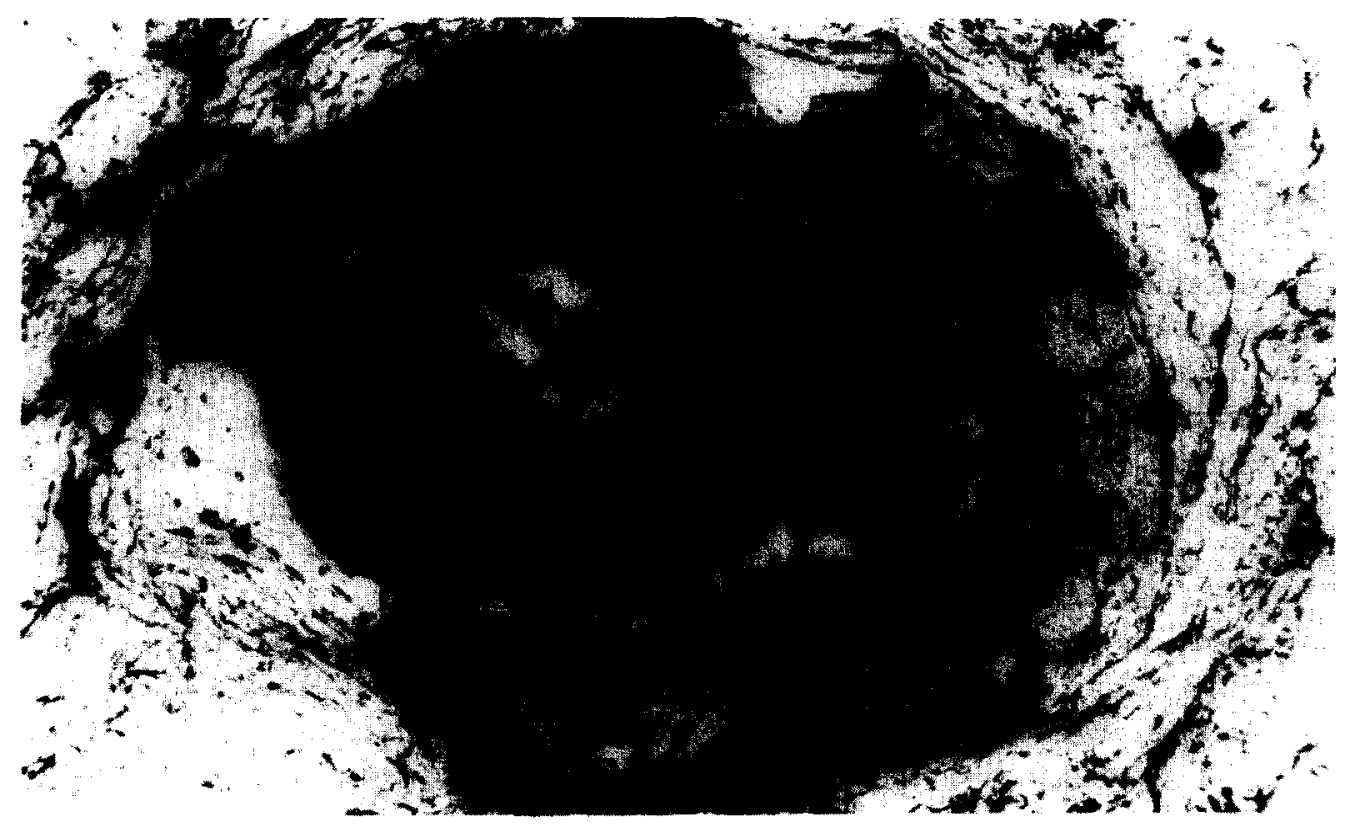

Fig. 2. Spiralling inclusion pattern in strongly paracrystalline-rotated garnet from a calcareous micaschist, Northern Adula region, Switzerland. 
plicitly made in these models in that they are in fact two-dimensional and only consider passive markers that intersect the shear plane at right angles to the shear direction. In such cases, it follows automatically that the true rotation axis of a rotated object like a porphyroblast should coincide with the intersection of the internal fabric and the external foliation. However, if a marker plane such as the foliation is considered to be truly passive, there is no reason to exclude initial orientations of a marker plane intersecting the shear plane oblique or even parallel to the shear direction. In such cases, the intersection of the internal fabric of a rotated porphyroblast and the external foliation defines an apparent rotation axis and the true angle between the internal fabric and the external foliation will only represent an apparent rotation angle which, inter alia, should not be confused with a reduced angle due to the cut effect in an oblique section. The following analysis serves to explore the effects of general initial orientations of the foliation on the apparent rotation axis and rotation angle of porphyroblasts affected by postcrystalline rotation, and aims to set a few simple rules to decide whether or not an apparent rotation axis can be interpreted as the true axis of rotation.

\section{The effect of initial orientation of the foliation}

A number of assumptions underlie the following geometrical approach. The foliation is thought to act as a material plane behaving passively in response to the imposed deformation. The relation between the amount of rotation of a porphyroblast and the magnitude of the strain is considered to be the same as that theoretically derived by Ghosh and Ramberg (1976) for rigid ellipsoidal bodies embedded in an isotropic viscous medium. Furthermore, in the case of nonspherical objects, one of the symmetry axes of the objects is thought to be parallel to the shear plane and perpendicular to the shear direction, and hence parallel to the true rotation axis. The deformation history is strictly homogeneous simple shear.

We will consider two different cases. One case includes all initial orientations of passive marker planes intersecting the shear plane parallel to the shear direction. Such marker planes maintain their initial orientation throughout the imposed simple shear deformation. The other case includes marker planes intersecting the shear plane in any direction oblique to the shear direction. These marker planes progressively rotate towards the shear plane with increasing strain.

The symbols used in the text are explained in Notation 1 (p. 283).

Case 1: passive marker parallel to the shear direction

Consider simple shear deformation which affects a volume of material containing a passive marker inclined at the shear plane and parallel to the shear direction (Fig. 3). Imagine a spherical porphyroblast which has grown in the matrix prior to deformation and has included the passive marker as a planar internal fabric. With increasing strain this porphyroblast will rotate about its true rotation axis $R$ according to (Rosenfeld, 1970; Ghosh and Ramberg, 1976):

$\dot{\Omega}=\frac{1}{2} \dot{\gamma}$

which results in an angle of rotation $\Omega$ for any given magnitude of shear strain $\gamma$. The internal fabric passively rotates with the porphyroblast about the $R$ axis. The apparent rotation axis $r$, defined by the intersection of the foliation and the internal fabric, progressively changes its orientation with increasing $\gamma$ along the stationary marker plane (Fig. 3a). A representation on a stereographic projection allows determination of the angle between the internal fabric and the marker plane, that is to say, of the apparent rotation angle $\boldsymbol{\theta}$. From inspection of Fig. 3 it follows that this apparent rotation angle has to vary between zero, for zero shear strain on any $360^{\circ}$ revolution about $R$, and a maximum for true rotations of $180^{\circ}$, $540^{\circ}$, etc., with $\theta_{\max }$ depending on the initial orientation of the marker plane with respect to the shear plane. It will also be noted that for any initial inclination of the passive marker, the apparent rotation angle $\theta_{\gamma}$ will be smaller than the true angle of rotation $\Omega_{\gamma}$ about $R$. This is shown in Fig. 4, which illustrates the value of $\theta$ as a function of $\gamma$ for two initial orientations of the 

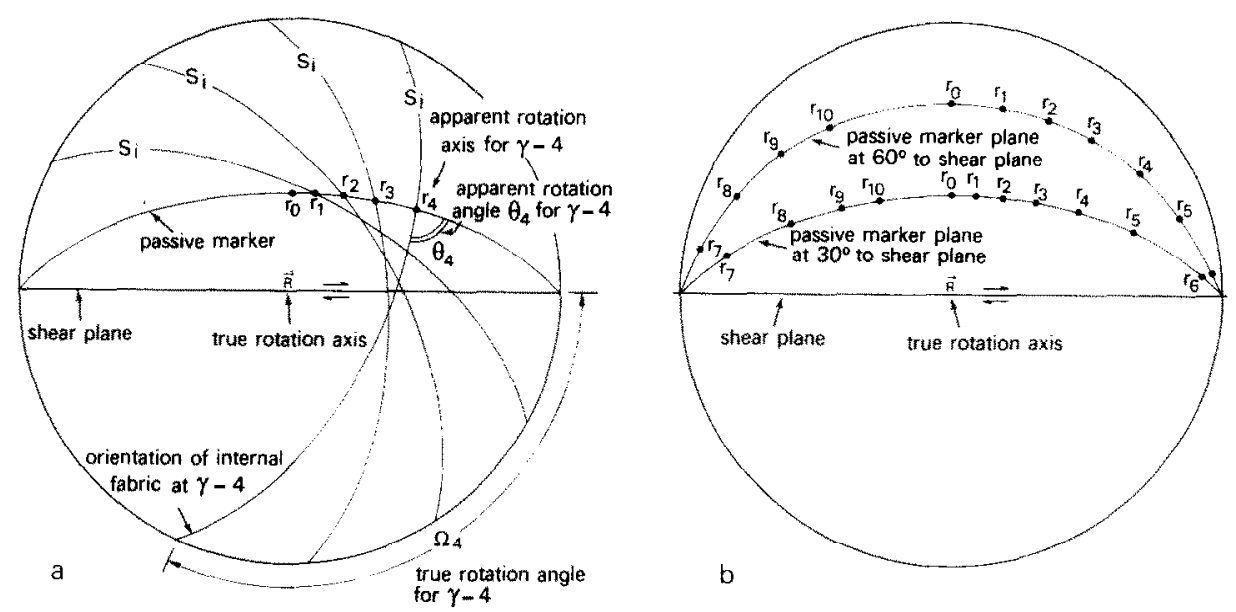

Fig. 3. a. Stereoplot showing changing orientation of apparent rotation axes with increasing shear strain of an ideally spherical porphyroblast rotating according to $\dot{\Omega}=\frac{1}{2} \dot{\gamma}$.

b. Orientation of apparent rotation axes with increasing shear strain for two different initial orientations of the passive marker.

passive marker. In the case of spherical porphyroblasts, there is no way to deduce the influence of this effect of the initial orientation of the marker plane, and a section through such a model rock would probably lead to an erroneous estimate of $\gamma$.

However, imagine a number of nonspherical porphyroblasts with variable axial ratios and variable initial orientations. In strictly simple shear, all of these porphyroblasts will rotate with the same sense about the true rotation axis $R$ according to the equation given by Jeffrey (1922):

$\dot{\Omega}=\frac{\dot{\gamma}\left(A^{2} \cos ^{2} \Omega_{0}+\sin ^{2} \Omega_{0}\right)}{A^{2}+1}$

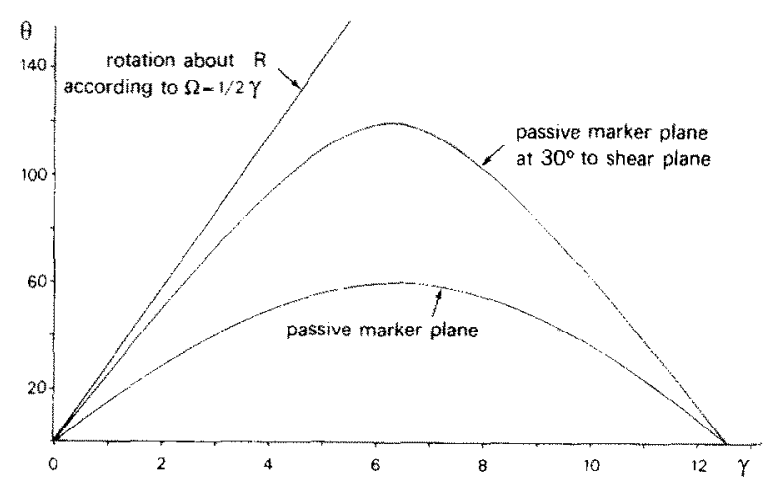

Fig. 4. Variation of the angle between internal and external foliation (apparent rotation angle) as a function of $\gamma$ for the two initial orientations of the passive marker in Fig. $3 b$. in which $A$ represents the axial ratio of a porphyroblast, and $\Omega_{0}$ the initial orientation of the long axis with respect to the normal to the shear plane. From this equation it follows (Ghosh and Ramberg, 1976) that during simple shear any nonspherical porphyroblast, with its long axis instantaneously within $45^{\circ}$ of the shear plane, will rotate more slowly than a spherical porphyroblast, while nonspherical porphyroblasts, with their long axes instantaneously at larger angles to the shear plane, will rotate faster than spherical porphyroblasts. As a result, variable angles of true finite rotation $\Omega$ are to be expected in the case of nonspherical porphyroblasts with variable axial ratios and variable initial orientations (Gosh and Ramberg, 1976) and, in particular for low values of $\gamma$, specific initial orientations of nonspherical porphyroblasts with specific axial ratios will produce minimum and maximum values of true rotation $\Omega_{\min }$ and $\Omega_{\max }$. For these low values of $\gamma$, the rotation angle $\Omega_{\gamma}$ of a spherical porphyroblast will be intermediate between these minimum and maximum values. Concomittantly, the apparent rotation axes spread over a sector containing $r_{\gamma}$ and limited by the orientation of $r_{\min }$ and $r_{\max }$, which are the apparent rotation axes of porphyroblasts rotated over $\Omega_{\min }$ and $\Omega_{\max }$, respectively.

This geometrical effect is qualitatively illustrated in Fig. 5. For large values of $\gamma, \Omega_{\gamma}$ eventually becomes larger than any true rotation 


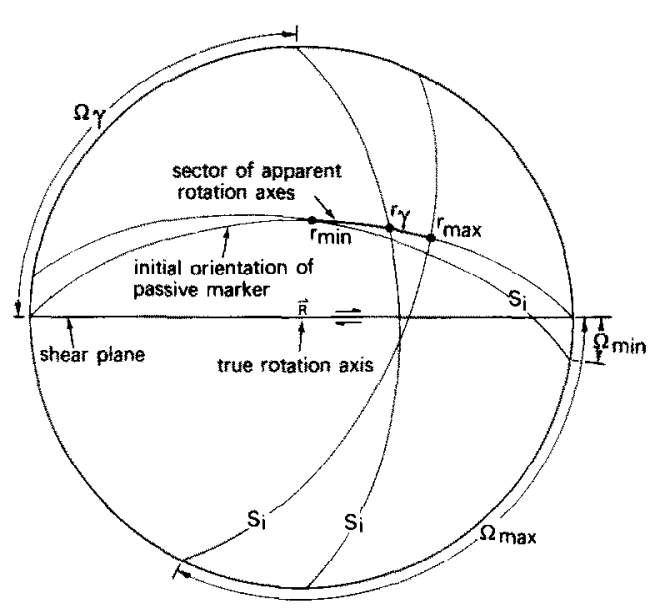

Fig. 5. Stereoplot showing sector of apparent rotation axes of nonspherical porphyroblasts. See text for further explanation.

angle of a nonspherical porphyroblast. This results from the fact that elongate porphyroblasts rotate very slowly through the shear plane with increasing $\gamma$. The sector of possible $r$ axis orientations is then bounded by the orientations of $r_{\text {min }}$ of specific, highly nonspherical porphyroblasts, and $r_{\gamma}$ of the spherical porphyroblasts. With small initial angles between the marker plane and the shear plane, the sector of possible $r$ axis orientations will be relatively small for low values of $\gamma$. For larger initial angles, the sector of $r$ axes rapidly increases with $\gamma$. As a consequence, though all porphyroblasts rotate forward and the marker plane is stationary, apparent forward and backward rotations are to be expected in any section through such a model rock that intersects the foliation within this sector of $r$ axes. However, the variable orientations of the apparent rotation axes in such a case is diagnostic to demonstrate the effect of the initial orientation of the marker plane with respect to the simple shear geometry.

Case 2: passive marker oblique to the shear direction

We consider again simple shear deformation which affects a volume of material, in this case containing a passive marker oriented such as to intersect the shear plane oblique to the shear direction. Such marker planes rotate asymptotically towards the shear plane with increasing strain (Fig. 6). A spherical porphyroblast grown prior to deformation includes the initial orientation of the passive marker, and during simple shear this planar internal fabric passively rotates about the true rotation axis $R$ according to eqn. (1). For any value of $\gamma$, the apparent rotation axis $r_{\gamma}$ is defined by the intersection of the internal fabric, rotated over $\Omega_{\gamma}$ about $R$, and the external fabric for that $\gamma$ (Fig. 6a). Three initial orientations of a passive marker have been studied and the results are presented in Fig. $6 \mathrm{~b}$. With increasing strain, the $r$ axes approach the shear plane along a conspicu-
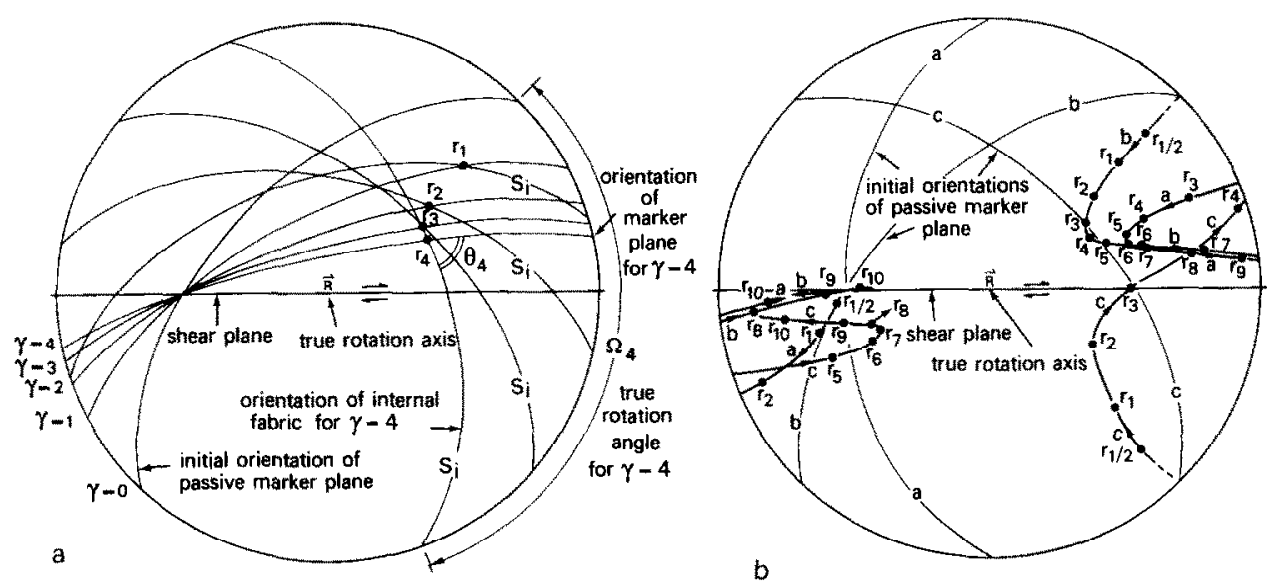

Fig. 6. a. Stereoplot showing changing orientation of apparent rotation axes of ideally spherical porphyroblasts rotating as a result of simple shear, in the case of a nonstationary passive marker. b. "Wandering paths" of apparent rotation axes with increasing shear strain for three different initial orientations of the passive marker. 
ous wandering path, primarily determined by the initial orientation of the marker plane. As in case 1 , the apparent rotation angle $\theta_{\gamma}$ is invariably smaller than the angle of true rotation $\Omega_{\gamma}$ (Fig. 7) and, irrespective of $\gamma$, never exceeds a maximum value that depends on the initial orientation of the marker plane. In any section through such a model rock with prekinematic spherical porphyroblasts, the use of $\theta_{\gamma}$ would again lead to underestimated values of $\gamma$.

Variable magnitudes of $\Omega$ arise in the case of nonspherical porphyroblasts that rotate according to eqn. (2). For a given value of $\gamma$, a sector of possible rotation axes results from all possible intersections of passively rotated internal fabrics with the passive marker for that $\gamma$ (Fig. 8). In contrast to case 1 , there is always an absolute minimum value of $\theta$, not equal to zero, which results from the rotation of the passive marker plane towards the shear plane with increasing strain, and therefore depends on the magnitude of $\gamma$ and on the initial orientation of the marker plane. This minimum value of $\theta$ occurs in porphyroblasts with their $r$ axes perpendicular to the true rotation axis $R$. Moreover, the apparent rotation angle is bound by a maximum, which for low strains occurs in specific nonspherical porphyroblasts, rotated over $\Omega_{\max }$ about $R$ (see Fig. 8). With increasing strain, an absolute maximum value of $\theta$ occurs in porphyroblasts with their $r$ axis again perpendicular to $R$, and with steadily increasing $\gamma$, minimum and maximum

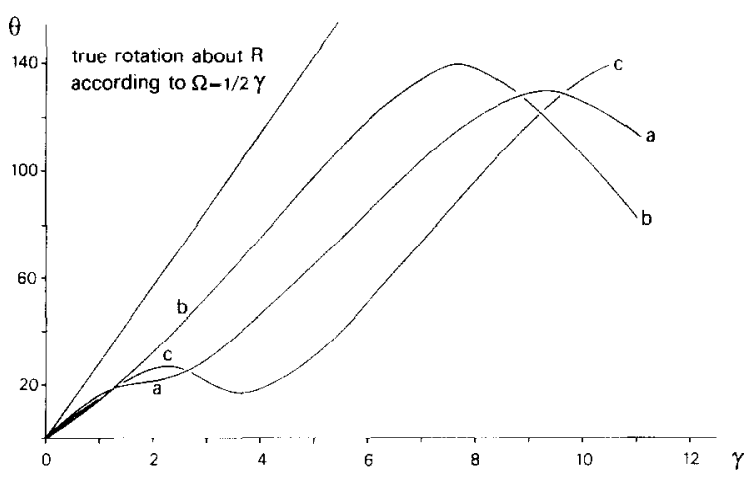

Fig. 7. Variation of the apparent rotation angle of spherical porphyroblasts with shear strain for the three initial orientations of the passive marker in Fig. 6 b.

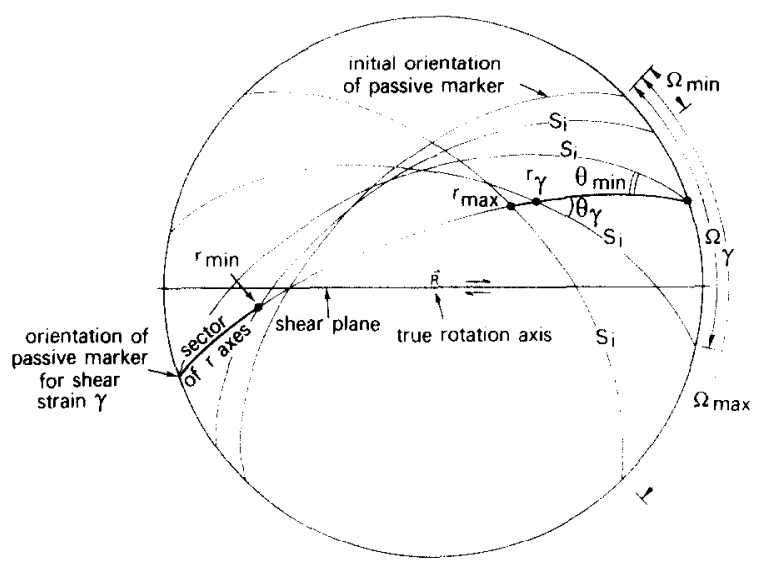

Fig. 8. Stereoplot showing sector of apparent rotation axes of nonspherical porphyroblasts in the case of the initial orientation in Fig. 6a. See text for explanation.

values of $\theta$ alternate for those special $r$ axes orientations. Owing to the gradual rotation of the passive marker towards the shear plane, subsequent minimum maximum values of $\theta$ increase asymptotically towards limiting values of $\theta_{\min }$ and $\theta_{\max }$ for infinite shear strain.

Apart from possible effects of the rate of rotation of the passive marker relative to that of the rotating porphyroblasts, the variable orientations of apparent rotation axes of nonspherical porphyroblasts will again lead to apparent forward and backward rotations in any section intersecting the foliation in the sector of $r$ axes.

\section{Apparent double rotations in paracrystalline porphyroblasts}

On the basis of the above analysis, one interesting point can be made regarding inclusion patterns in paracrystalline rotated porphyroblasts. As pointed out by Schoneveld (1977), the matrix bordering a rotating porphyroblast in a foliated rock is essentially made up by two types of domains: a domain (1) with unoriented coarse minerals defining pressure shadows adjacent to the porphyroblast, and a domain (2) of commonly finer grained minerals with a strong shape fabric parallel to the foliation in the rock. During growth of the rotating porphyroblast, this shape fabric is continuously included. From the preceding geometrical analysis, it follows that any segment of 
the included fabric from relic domain 2 will have an orientation such as to intersect the foliation along an apparent rotation axis. This axis is the apparent axis for a rotating passive marker at the value of the shear strain accumulated since the inclusion of that segment. As the amount of finite true rotation decreases from the core of a paracrystalline porphyroblast towards the rim, we may for the matter of this analysis consider the porphyroblast as being build up by segments of domain 2, each of which includes an apparent rotation axis. To the knowledge of the author, only Rosenfeld (1970) has referred to possible effects of such a change of orientation of (apparent) rotation axes; he presented a ring model to explain the three-dimensional shape of the resulting inclusion fabric (Rosenfeld, 1970, plate 11). As outlined above, the changing orientation of apparent rotation axes defines a sector, and any section through this sector will show apparent forward and backward rotations depending on the amount of true rotation. In the case of a paracrystalline porphyroblast, these apparent forward and backward rotations may be recorded as an inversion of the apparent sense of rotation.

This phenomenon is illustrated in Fig. 9, which shows two sections through a model porphyroblast with a linear growth rate proportional to the paracrystalline shear strain rate, and constructed for the initial orientation of the passive marker shown in Fig. 3a. The two sections are perpendicular to the foliation, one being parallel and one perpendicular to the shear direction. The section parallel to the shear direction shows the wellknown snowball pattern of spiralling inclusion trails. In the section perpendicular to the shear direction, a conspicuous reversal of the apparent sense of rotation is produced by the changing orientations of subsequent apparent rotation axes from the core of the porphyroblast towards the rim. Such microstructures have previously been explained by Dixon (1976) and Ghosh and Ramberg (1978) as being due to the effect of the relative rotation rate of the porphyroblast with respect to the rotating foliation. Although the author fully accepts this explanation for apparent reversals in paracrystalline rotated porphyroblasts, an initially oblique orientation of the foliation with respect to the simple shear geometry is equally capable of producing such structures. Mutually perpendicular sections are needed in such a case to elucidate which explanation applies in a particular rock.

\section{Discussion and conclusions}

The above geometrical approach leads to some qualitative conclusions on the significance of the

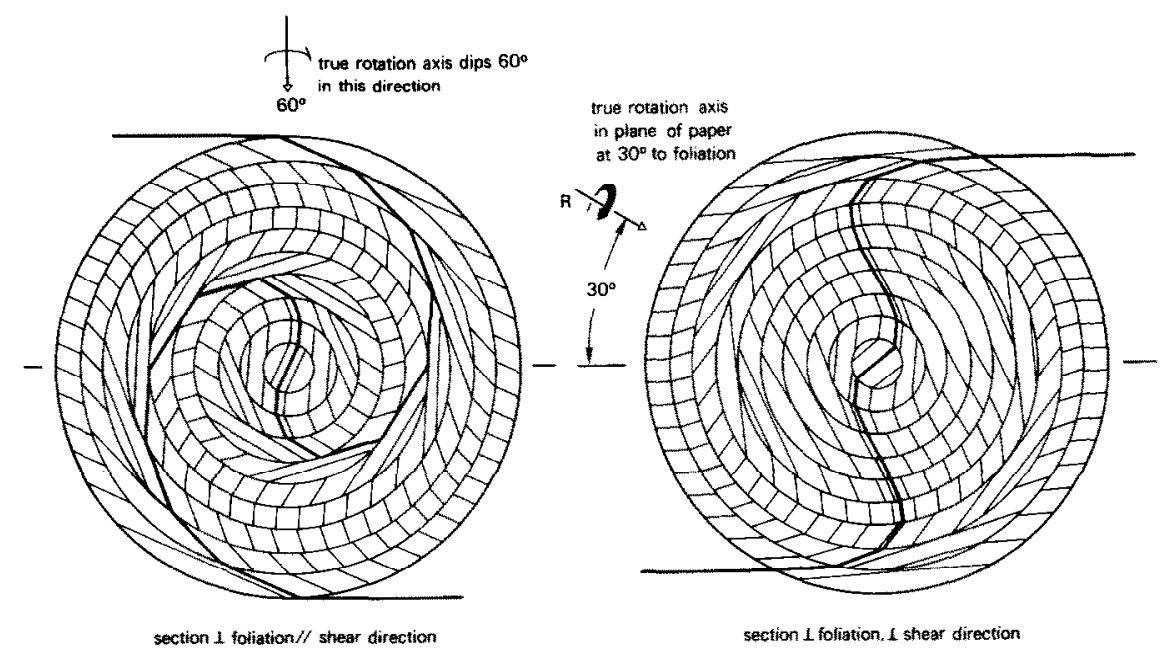

Fig. 9. Two sections through a paracrystalline rotated model porphyroblast grown in a matrix with a passive marker initially oriented as in Fig. 3a. 
apparent rotation axis and rotation angle of postcrystalline rotated porphyroblasts when the foliation, considered to behave as a passive marker plane, is obliquely oriented with respect to the geometry of a homogeneous simple shear:

(1) For any value of the shear strain $\gamma$, the apparent rotation axis makes a distinct angle with the true axis of rotation. This angle varies with the amount of true rotation, hence with $\gamma$, and is invariably larger than or equal to the initial angle between the true rotation axis and the passive marker at the very onset of deformation. The changing orientation of the apparent rotation axis with shear strain may result in inclusion patterns in paracrystalline rotated porphyroblasts that show apparent reversals in specifically oriented sections.

(2) As the orientation of the apparent rotation axis varies with the amount of true rotation, variable $r$-axis orientations are to be expected in the case of nonspherical porphyroblasts with variable aspect ratios and variable initial orientations. This leads to a sector of possible $r$ axes, instead of a distinct single orientation of apparent axes.

(3) The apparent rotation angle is invariably smaller than the corresponding true angle of rotation. The apparent angles vary between either zero or a distinct minimum $\theta_{\min }$, and a maximum value $\theta_{\max }$ in the case of nonspherical porphyroblasts, and both minimum and maximum values depend on the initial orientation of the passive marker and the magnitude of the strain.

The above analysis only applies if, during the deformation leading to porphyroblast rotation, the pre-existing foliation behaves as a passive marker. Many foliations in metamorphic tectonites, in particular those developed in micaschists and in mica-rich quartzites, are unlikely to behave mechanically passive. A strong mechanical anisotropy obliquely oriented with respect to the geometry of an imposed simple shear deformation will probably lead to partitioning of the bulk simple shear flow into slip along the anisotropy, coaxial stretching along the anisotropy, and bulk rotation or spin. Therefore, at the microscale in a rock to which such a situation applies, one may expect that the foliation will behave as a plane of shear, which reduces the above analysis to a geometrically simpler case, included in studies such as those by Ghosh and Ramberg (1976). However, a mechanically passive behaviour of an early foliation can be envisaged in certain rocks, such as mica-bearing quartzites, in which isolated flakes of oriented mica define an earlier tectonic fabric, but in which deformation is entirely taken up by flow in the quartzite mass.

A quantitative study, although possible, has been avoided here because of its limited applicability, which results particularly from the restriction made to the case of homogenous simple shear. Many natural deformations will likely have a simultaneous pure shear component, leading to distinct effects on the rate of rotation of the passive marker and on the rotational behaviour of nonspherical porphyroblasts. For a given ratio of the rates of pure shear versus simple shear, porphyroblasts with aspect ratios beyond a critical value will tend to orient themselves in stable orientations (Ghosh and Ramberg, 1976). This effect does not preclude the above qualitative conclusions, but may have serious effects on the values of the apparent rotation angles.

In any case, whether or not a component of pure shear is involved in the deformation, the above conclusions may help to identify the effects of an initial orientation of the pre-existing foliation oblique to the geometry of the deformation. The variable $r$-axis orientations of nonspherical porphyroblasts are indicative of such an initial orientation. Inspection of thin sections parallel to the foliation is probably the most efficient way to establish any variability of $r$-axis orientations. However, the argument should be carefully restricted to those cases in which other observations preclude the possibility of a multi-stage deformation history involving two or more rotations about different rotation axes. Alternatively, consistency of apparent rotation axes of nonspherical porphyroblasts with variable apparent rotation angles unambiguously indicates that the apparent axes coincide with the true axes of rotation, and that the apparent angles can be safely interpreted as true rotation angles. 


\section{Acknowledgements}

Thanks are due to Hans de Bresser and Cees Passchier for their constructive criticism and correction of the manuscript.

\section{Notation 1}

Explanation of symbols used in the text

\begin{tabular}{|c|c|}
\hline$\gamma$ & shear strain \\
\hline$\Omega$ & true angle of rotation \\
\hline$\Omega_{0}$ & $\begin{array}{l}\text { initial angle between long axis of porphyroblast } \\
\text { and normal to the shear plane }\end{array}$ \\
\hline $\boldsymbol{\Omega}_{\gamma}$ & $\begin{array}{l}\text { true rotation angle of porphyroblast rotated } \\
\text { according to } \Omega=\frac{1}{2} \dot{\gamma}\end{array}$ \\
\hline$\Omega_{\min }, \Omega_{\max }$ & $\begin{array}{l}\text { minimum and maximum angle of true rotation of } \\
\text { nonspherical porphyroblast for given value of } \gamma\end{array}$ \\
\hline$\theta$ & apparent rotation angle \\
\hline$\theta_{\gamma}$ & $\begin{array}{l}\text { apparent rotation angle of spherical porphyro- } \\
\text { blast rotated according to } \Omega=\frac{1}{2} \dot{\gamma}\end{array}$ \\
\hline $\begin{array}{l}\theta_{\min }, \theta_{\max } \\
R\end{array}$ & $\begin{array}{l}\text { minimum and maximum values of } \theta \text { for given } \gamma \\
\text { true rotation axis }\end{array}$ \\
\hline$r$ & apparent rotation axis \\
\hline$r_{\gamma}$ & $\begin{array}{l}\text { apparent rotation axis of spherical porphyroblast } \\
\text { rotated according to } \Omega=\frac{1}{2} \dot{\gamma}\end{array}$ \\
\hline$r_{\min }, r_{\max }$ & $\begin{array}{l}\text { apparent rotation axis of nonspherical porphy- } \\
\text { roblasts rotated over } \Omega_{\min }, \Omega_{\max } \text { for a given value } \\
\text { of } \gamma\end{array}$ \\
\hline$s_{\mathrm{i}}$ & internal fabric in porphyroblast \\
\hline
\end{tabular}

\section{References}

Dixon, J.M., 1976. Apparent "double rotation" of porphyroblasts during a single progressive deformation. Tectonophysics, 34: 101-115.
Ghosh, S.K. and Ramberg, H., 1976. Reorientation of inclusions by combination of pure shear and simple shear. Tectonophysics, 34: 1-70.

Ghosh, S.K. and Ramberg, H., 1978. Reversal of the spiral direction of inclusion-trails in paratectonic porphyroblasts. Tectonophysics, 51: 83-97.

Jeffery, G.B., 1922. The motion of ellipsoidal particles immersed in a viscous fluid. Proc. R. Soc. London, Ser. A, 102: 161-179.

Powell, D. and Treagus, J.E., 1967. On the geometry of S-shaped inclusion trails in garnet porphyroblasts. Mineral. Mag., 36: 453-456.

Powell, D. and Treagus, J.E., 1970. Rotational fabrics in metamorphic minerals. Mineral. Mag., 37: 801-814.

Ramsay, J.G., 1962. The geometry and mechanics of formation of 'similar' type folds. J. Geol., 70: 309-327.

Rosenfeld, J.L., 1970. Rotated garnets in metamorphic rocks. Geol. Soc. Am., Spec. Pap., 129: 102 pp.

Schmidt, W., 1918. Bewegungsspuren in Porphyroblasten Kristalliner Schiefer. Sitzungsber. Akad. Wiss. Wien, Abt. 1, 127: 293-310.

Schoneveld, C., 1977. A study of some typical inclusion patterns in strongly paracrystalline-rotated garnets. Tectonophysicis, 39: 453-471.

Schoneveld, C., 1979. The geometry and the significance of inclusion patterns in syntectonic porphyroblasts. Ph.D. Thesis, State University Leiden, Leiden, $125 \mathrm{pp}$.

Spry, A., 1969. Metamorphic Textures. Pergamon, London, $350 \mathrm{pp}$.

Zwart, H.J., 1962. On the deformation of polymetamorphic mineral associations and its application to the Bosost area (Central Pyrenees). Geol. Rundsch., 52: 38-65. 